\title{
21
}

\section{A framework for cooperation in virtual enterprises}

\author{
L. M. Camarinha-Matos, C. Lima \\ New University of Lisbon - Faculty of Sciences and Technology \\ Quinta da Torre - 2825 Monte Caparica - Portugal \{cam, \\ cpl\}@uninova.pt
}

\begin{abstract}
The concept of virtual enterprise challenges the way industrial production systems are planned and managed. The materialization of this paradigm, although enabled by recent developments in communication technologies, computer networks, and cooperative systems still requires a full understanding of the processes of interaction between companies as well as the design and implementation of open support platforms. The reengineering of activities for a networked operation and the implantation methodologies considering not only the technical but also the socio-organizational aspects must be also taken into account. This paper describes the current approach being developed by the Esprit project PRODNET II $^{1}$, which aims to design and develop an open platform to support industrial virtual enterprises with special focus on the needs of small and medium enterprises (SMEs).
\end{abstract}

\section{Keywords}

Virtual Enterprises, Workflow management systems, Cooperative Systems, Information Interchange

\footnotetext{
'This work is based on the ongoing activities of the European Union funded project entitled PRODNET II, the ESPRIT project 22647. It involves partners from Europe (CSIN, New University of Lisbon, ESTEC, Miralago, University of Amsterdam, Lichen Informatique, CIMIO, Uninova) and Latin America (Federal University of Santa Catarina and Herten), including Research Institutes, Engineering companies and end users. (Home page http://www.uninova.pt/ prodnet.)
} 


\section{INTRODUCTION}

Recent developments in various complementary areas such as communications, computer networks, cooperative systems, transportation logistics, and advanced information management, represent important enabling factors for emergence of new approaches to organize and manage manufacturing systems. In this context, networking of enterprises and outsourcing or subcontracting are particularly strong tendencies in terms of organization of the global manufacturing and supply chain processes.

The concept of virtual enterprise (VE), as a temporary alliance of enterprises that come together to share skills and resources in order to better respond to business opportunities and whose cooperation is supported by computer networks, challenges the way industrial production systems are planned and managed. The advances in logistics, reducing the transportation costs and increasing the reliability of the supply flow, changed the meaning of the physical distance between the VE members. The main concerns are now related to the implementation of a real Electronic Data Interchange supporting the reliable exchange of orders, product models, catalog of products, product quality information and coordination messages.

In spite of the mentioned advances, there is a need for faster and more reliable communication infrastructures, as well as for a framework for flexible interoperation and cooperation between heterogeneous and autonomous nodes.

This paper describes the current approach being followed by the Esprit project PRODNET II, which aims to design and develop an open platform to support industrial virtual enterprises with special focus on the needs of small and medium enterprises (SMEs). PRODNET II is defining process to allow a gradual migration of SMEs towards a VE environment, in such a way to minimize undesirable impacts (both technical and social) on the internal side of a company. The assumption is that although companies may want to join a VE on a voluntary basis, they want to monitor and control their inclusion and participation in that VE, based on their own rules.

In order to support openness, PRODNET II tries to adopt, as much as possible, international standards, a flexible, reliable and configurable coordination management, guaranteeing the privacy and autonomy of participating companies. Therefore, the PRODNET II architecture is based on STEP, EANCOM, WfMC and SET protocol. A detailed description of the PRODNET II architecture can be found in (Camarinha-Matos, 1997a 1997c, Afsarmanesh, 1997a, 1997b). This paper is mainly focused on the information flows and is organized as follows. Section 2 gives a general overview of the PRODNET II platform and architecture. Section 3 presents the classes of information and standards adopted by PRODNET II. Section 4 discusses the PRODNET approach to orders management, with special emphasis on the incomplete and imprecise orders. Management and control inside the PRODNET II platform is discussed in the section 5. Open questions and conclusions are presented in the section 6. 


\section{THE PRODNET PLATFORM}

Due to many factors (social, market strategy, etc.) is foreseeable to find different levels of interoperation between companies in a VE environment. Despite the classical way of make business individually is becoming obsolete, this not means that companies will start working together in a hundred percent trustworthy way. On the contrary, companies are start working cooperatively sharing only a minimal set of information. They have to be convinced that public computer networks can offer privacy and safety. They are waiting for seeing the results. If the benefits are real, companies might visualize a new horizon in terms of business strategy. Which means, they probably will be willing to share more information, even resources, in a VE environment. This is why PRODNET architecture relies on a gradual movement towards the VE environment.

In a first level, any company will be able to integrate a VE environment based on its internal rules. This guarantees a complete independence and autonomy of the company. However, if a company wants to share more and be more integrated to the environment, PRODNET supports it in a second level of integration. Finally, the ultimate goal is to support a VE environment fully integrated, in which companies already learnt to trust each other and they also know what to expect from a VE, in terms of profits.

\subsection{PRODNET Architecture}

To support this environment and coping with the legacy systems in enterprises, PRODNET II proposes an infrastructure including two main modules (CamarinhaMatos, 1997b, 1997c): the Internal Module and the Cooperation Layer (PCL). The Internal Module represents the autonomous unit of a particular company. It includes the complete structure of the company's information (databases, information systems, etc.) and all the internal decision making processes / enterprise activities (internal PPC and engineering systems). The Cooperation Layer contains all the functionalities for the inter-connection between the company and the entire net. It represents the communication and coordination role and works as the interlocutor of the company within the net (Figure 1). For each node of a VE network, PRODNET proposes the following architecture:
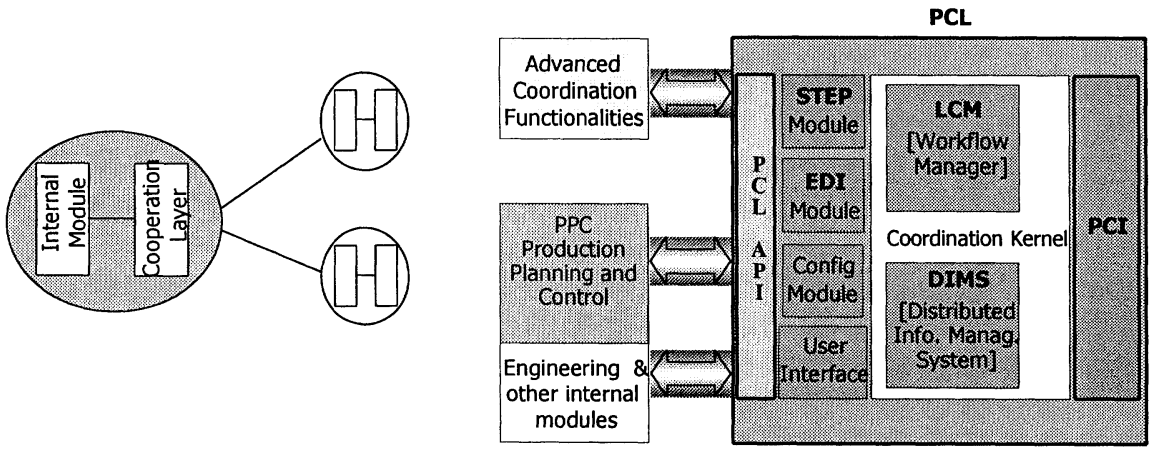

Figure 1 PRODNET Reference Architecture. 
Where the main components are the following:

PPC - Production Planning and Control System. This is the most important component of the internal module of a VE node. It includes functions as: i) Industrial Logistics Management: Orders flow management, Product data management, Sales Forecasts handling, Actual Requirements Planning; ii) Master Production Scheduling; iii) Production Control; iv) Quality Control / Tracking; and v) Industrial Costing.

DIMS - Distributed Information Management System. The Distributed Information Management Subsystem in the PRODNET Cooperation Layer is responsible to model and manage all cooperation support information (Afsarmanesh, 1997a, 1997b).

EDI Module. This module is responsible for receiving and formatting ordersrelated messages in EDIFACT format. Among its functionalities, it will check / parse EDIFACT syntax (for various versions of the standard), check for completeness of messages contents, and generate appropriate formats for sending out EDI messages. It will also detect and extract EDI messages embedding information represented in other formats, such order-associated STEP specifications.

STEP Module. The STEP module's function is to handle the technical product data used within PRODNET. Ideally all product data should be exchanged in STEP format. The STEP services provided to PRODNET will allow for the transmission and reception of STEP files that have been clear text encoded according to a defined schema; as described in Part 21 of the STEP standard. It should also be possible to query that STEP data held within PRODNET by the usage of the Standard Data Access Interface, SDAI, or via a SQL interface.

$P C I$ - PRODNET Communication Infrastructure. This module is responsible for handling all communications with the other nodes in the network. It includes functionalities such as: Selection of communications protocol and channels; Basic communications management; Privacy mechanisms (cryptography); and Secure message communication channels between nodes.

Configuration and User Interface. The PRODNET platform is intended to support a large diversity of enterprises and interconnection modes. This means a large heterogeneity in terms of available / installed services and desired management procedures. Therefore it is necessary to specify the desired cooperation behavior in an explicit plan (each enterprise has to define its particular activity flow plan) that will be "executed"/controlled by the Local Coordination Module.

\subsection{Information Handling and Coordination Kernel}

PCL receives all messages coming from and to be sent to the VE environment. Every message might flow among PCL modules and it has to be processed following a particular sequence of actions, using the data associated to it. A clear separation between data and control was adopted inside PCL, based on two modules responsible for coordination and data management: LCM and DIMS. LCM, a workflow engine based on the WfMC concepts, is the responsible for the management/control of all messages inside PCL. DIMS, a distributed federated 
database module, is the component responsible for managing all data flowing through PCL. These aspects will be discussed later, in the section 5 .

A strong cooperation between LCM and DIMS is also the basis to the hierarchical workflow approach adopted by PRODNET II to support an incremental move of SMEs towards the VE environment. In the first phase of this incremental move, a company starts working within a VE according to its internal rules. This means a human-supervised control of what to share and monitor what is exactly happening in terms of VE transactions. On a later stage, depending on the achievement of higher levels of trust between companies involved in a VE, some of these processes can be progressively automated. DIMS supports the sharing of information among partners, whilst LCM provides control and monitoring of the company participation in a VE.

\section{INFORMATION INTERCHANGE}

There are several classes of information to be exchanged between companies in a VE. The extent of this interchange depends on the level of cooperation between companies. PRODNET is supporting the following classes:

\subsection{Business-related Information}

Orders-related information is the basis of the business-related information exchanged between partners in a VE, since the relationships between client and supplier are mainly based on orders. Orders-related information includes the order data itself but also quality information about the product ordered. Sometimes product models and catalogue of products are exchanged as additional information to an order.

Quality information is becoming more and more important in the commercial relationships. The customers are demanding better products, in all senses. A client may ask for quality certificates from its suppliers. The suppliers do the same to their sub-contracted partners and this process goes further and further, along the whole supply chain.

PRODNET II provides EDI-related services to support the exchange of commercial information. EDI provides trading partners with an efficient business tool for the automatic transmission of commercial data from one computer system directly to another. Some companies have been practicing EDI on the basis of proprietary formats since the late 1960's (EAN, 1996). However, an EDI system was not a cheap one and it used to take a long time before start running. By the mid-1980's the development of EDI standards began taking shape within the United Nations Economic Commission for Europe. In 1987, the syntax of this common business language, known today under the acronym of UN/EDIFACT ${ }^{2}$, was approved as an ISO standard. PRODNET EDI-related services are being based

\footnotetext{
${ }^{2}$ UN-EDIFACT is the United Nations Rules for Electronic Data Interchange for Administration, Commerce and Transport. It comprises a set of standards internationally agreed, directories and guideline for the electronic interchange of structured data and in particular the related documents to trade in goods and service.
} 
on the UN/EDIFACT standard and developed by the Lichen Informatique company.

Nowadays, the EDI standards, although not yet easy to implement, are becoming more accredited and popular. Through the use of EDI message standards such as EANCOM, data may be communicated quickly, efficiently and accurately irrespective of the users' internal hardware and software equipment (EAN, 1996). Moreover, the competition against the HTML-based forms in the WWW world is forcing a new commercial vision of EDI, leading to an effort to integrate WWW and EDI. PRODNET II follows this approach.

The EDI protocol can also be used support the exchange of catalogs of products. However, catalogs are traditionally based on pictures as well as multi-media information. Considering that the WWW world cannot be set aside from the PRODNET platform, PRODNET II is also analyzing the use of HTML forms to support the exchange of product catalogs.

\subsection{Engineering Information}

This class is based on the Product model information. Some orders can carry product-engineering information to better specification purpose. A client might want to order a new product or component and a product model is necessary to accurately define the requirements. A negotiation process related to a product design can be carried out supported by the interchange of these electronic product models. The STEP standard provides a normalized way to support the exchange of product models. It allows the expression in a uniform and complete way of the whole information required for a product during its life cycle, especially through the EXPRESS language. Thus, two companies working in the same industrial sector can model and exchange their products according to the Application Protocol (AP) defined for that sector.

PRODNET II provides services based on the STEP standard to support the exchange of product model in a VE, even if one partner does not have an internal CAD tool able to read STEP data. PCL supports the translation to/from STEP data from/to native CAD formats. PCL also supports the visualization of STEP models. Complementary STEP services are also going to be provided by PCL. The set of STEP related functionalities is developed by the CIMIO company.

\subsection{Privacy/Security and Authentication of Exchanged Information}

Reliable and safe information interchange is a key point in any VE. This is a strong requirement in order to convince SMEs that making business electronically through a public network can be efficient and trustable. Cryptography algorithms based on special techniques to assure privacy and security through public networks have been adopted, such as symmetric (private-key) and asymmetric (public-key) cryptography. Privacy can be guaranteed by the utilization of both symmetric and asymmetric cryptographic techniques.

Authentication is based on a digital signature, which allows the receiver to check if the message was changed. Besides that, it can be used to legally associate the 
message to the sender. The digital signature is also encrypted with the symmetric/asymmetric algorithms.

PRODNET II is offering a communication infrastructure enhanced with both privacy and authentication facilities. The ESTEC company and UNL are involved in this development.

In addition to these generic classes of information, there is also information related to the VE management that needs to be shared and managed. Examples of such information are: company profile, VE structure and access rights of its members, public and shared information, etc. The University of Amsterdam is the main responsible for the development of a distributed and federated information management infrastructure for VEs.

\section{ORDERS MANAGEMENT}

The electronic exchange of orders is a basic functionality to be provided by a VE supporting platform. Besides the exchange of the order itself, it is necessary to support its follow up in order to cope with, for example, delays in orders processing, temporary incapacity of a supplier, changes in not completed orders, the need to re-adjust delivery times, and so on. A client company might even want to know details about the manufacturing status of ordered products or components in order to prevent any difficulties for itself. Orders management is, therefore, one of the most important functionalities to be supported in a VE environment.

In the PRODNET II architecture it is assumed that efficient orders management is only possible if a company already has some PPC system which provides the company's internal orders management. Otherwise it seems unfeasible because the amount of information required to perform such management should not be supported/handled by a component like PCL. Thus, a basic requirement in order to accomplish the orders management is a strong interaction between PCL and PPC system. This implies some reengineering of the legacy PPC system in order to make it reactive to requests coming from the network and to make it able to understand the chosen EDI format.

PRODNET II is also dealing with a very challenging requirement in the orders management, that is the ability to support the flow - and the acceptance by suppliers - of incomplete and imprecise orders. This is mainly a consequence of the need of self-accommodation to rapidly changing markets. Incomplete and imprecise orders do not have all information about them defined before starting their production process. PRODNET approach to deal with this problem is discussed in the next sections.

\subsection{Classes of Orders}

PRODNET II considers three types of orders: Regular, Incomplete and Imprecise. There is no information vagueness (incomplete or imprecise data) in a regular order, which means all information related to that order is known. On the other hand, an incomplete order has at least one piece of data missing, whilst an imprecise order has at least one piece of data specified in a vague way. These two 
types of orders require a special handling process as the data required to fulfill the order will arrive incrementally. In spite of the incompleteness or vagueness of information, it is often possible for a company to start the production based on the available information as well as to propagate that vagueness to its suppliers.

\subsection{PPC requirements for orders management}

The PPC system is the component which embeds the knowledge to manage the orders-related messages. Inside the PPC, orders can go through several stages along the time, according to their status of accomplishment. Thus, it shall be possible to query the PPC in order to know what is exactly happening to every single client order at any moment. In PRODNET II an order life cycle includes the following main states:

- Launched : a new order is received;

- Confirmed: the order is confirmed, the production phase can start;

- Partially shipped: the product(s) required by the order is (are) partially shipped to the client, while the remaining part is still in production;

- Production: the order is in production;

- Fulfilled: the order execution is completed;

- Cancelled: the order is cancelled.

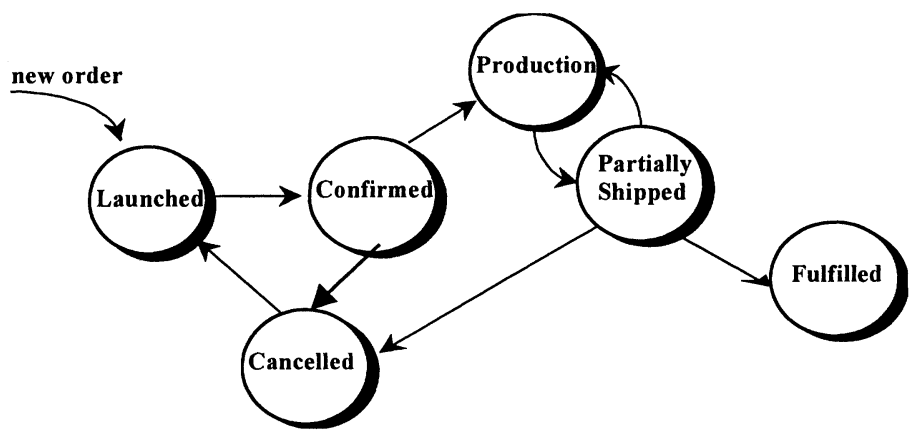

Figure 2 Order life cycle diagram.

Figure 2 illustrates this life cycle. The loop involving the states Production and Partially Shipped represents the situation in which part of the required product is ready to be delivered to the client as soon as it is ready, whilst the rest of the order remains in production.

An order model is divided in two parts (Figure 3). The first part is composed by the General Attributes such as delivery dates, status, quantities, customer identification, etc. The second part is composed by the Product Details: product identification, invariant characteristics, optional characteristics. One important concept related to the Product Details is the representation of a product using two different sets of attributes: the invariant and the variant attributes. This representation is the key idea behind the management of incomplete and imprecise orders, because it is always possible to start the production of a product using the invariant part. For example, a car always has wheels, doors, chassis, engine 
(invariant attributes). But the color and the number of doors are configurable parameters (variant attributes), which can be imprecisely or incompletely defined.

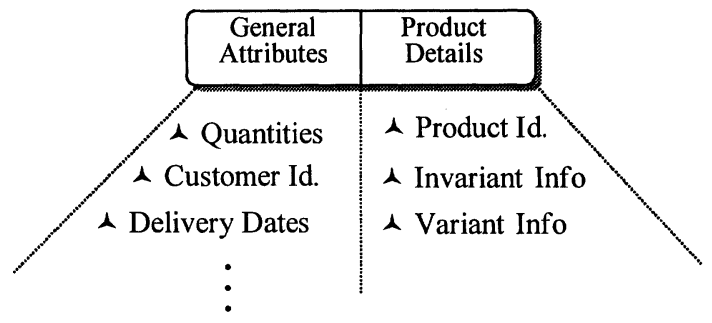

Figure 3 Order Model.

There are two types of orders, according to the Product Details part. These types depend on the way a company is placed in the market or the product it sells.

One type is related to products that do not have a major component. It is important to think in terms of the influence, on the final product, caused by imprecision/incompleteness in some of the product's attributes.

Let us consider, for example, an order for a car model $\mathrm{M}$, for which it is possible to specify particular features (optional characteristics), like the type of engine, the color, etc. An incomplete order does not include all required product details. An order could, for instance, simply specify a car model $M$. If an order like that is received, then it is possible to start the production of the chassis, the doors, etc., independently of the missing information about complementary attributes. New "orders" (or messages) are supposed to arrive in future to complement the missing attributes in the "original" order (these orders are not really new orders but additions to the original one). Thus they should be associated to the original one (logically merged). This process continues until all details about the required product have been specified.

An imprecise order is an order in which the value of some attribute is not missing but it is specified in a vague way. For instance, in the car example, the attribute color, in the initial specification, could say "dark color" or "one of blue, black, green". Only later on this attribute would get a specific (precise) value. Another example of vagueness could be on the quantity. An order could specify a tentative amount of between 100 and 120 units (to be confirmed later).

In a second type, the final product has a major component or raw material. In this case, it is possible to overcome the imprecision / incompleteness regarding the product by forcing a commitment to a precise quantity of that component. This type of contract is a common practice in the garment industry. For instance, when an order for suits is not precise in terms of quantities and other model-related details, it is common to establish a contract in which the client commits himself to use / consume a given amount of the main component (a particular kind of fabric) independently of the product details to be specified later. PRODNET II is considering the ability to handle both types, as an important requirement for the orders management. 


\subsection{PCL requirements to deal with orders}

The orders related messages will flow among VE partners coded in an EDI format. Thus, PCL has to provide the EDI services to support the exchange of all types of orders-related messages. Some services are of general purpose, such as creation, identification and parsing of message orders; other services are specific to support the management of incomplete and imprecise orders.

PCL adopted the EAN international standard for EDI communication (EANCOM) to support the message orders requirements. EANCOM is a detailed implementation guideline for the UN-EDIFACT standard messages. It provides clear definitions and explanations which allow trading partners to exchange commercial documents in a simple, accurate and cost effective manner. One class of standard messages available in EANCOM is Commercial Transaction Messages, which covers the general trading cycle from quotation to request to remittance advice. This class includes the Purchase Order set of messages which is related to the ordering process from a proposed order, subsequent changes and the eventual confirmation (relevant quantities, dates, location of delivery, etc.) (EAN, 1996). In this way, the PCL EDI-related services can cover all orders-related requests presented in the previous section, including the management of imprecise and incomplete orders.

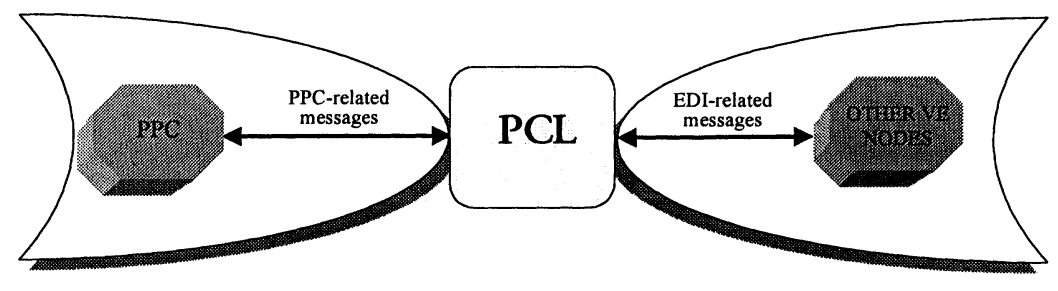

Figure 4 Relationship between PPC, PCL and the VE

Another aspect is related to the conversion from/to EANCOM/PPC internal formats. PCL can translate the VE messages to the appropriate PPC format and vice-versa. In order to make this task easier, the PPC database, developed by the CSIN company, adopted the EDI data definition (Figure 4).

\section{PCL INTERNAL MANAGEMENT/CONTROL STRUCTURE}

PCL management and control structure relies on two major aspects: configuration of the PCL behavior and coordination among PCL modules. The former is supported by the LCF module. The later requires a clear separation between the control itself and the data needed to support the control. PCL receives messages from both the company internal module and from other VE members. Each message is associated to a specific PCL service. For each message, PCL has to know what to do with it, what are the actions that should be performed in reaction to the message and how to manage the data involved in the message. 
Three PCL modules are involved in this process: Local Configurator Module (LCF), Local Coordinator Module (LCM) and Distributed Information Management System (DIMS). LCF supports the configuration of the PCL behavior. LCM is responsible for the coordination among PCL modules. DIMS is responsible for the data management and storage inside PCL.

\subsection{Configuration aspects}

The key point in the PCL configuration is the ability to support a gradual migration towards a VE environment operation. In a first level, PCL has to guarantee that the company's privacy, autonomy as well as its way of making business will be preserved as far as possible. This is provided by LCF module through a flexible configuration, based on a strong interaction with a human operator, representing the company needs. LCF deals with three classes of information. First, the configuration of the company environment is defined in the Company Profile. The PCL behavior is defined in the PCL Workflow Model. Finally, the configuration of the relationship between the company and the VE environment is defined in the $V E$ Network Directory.
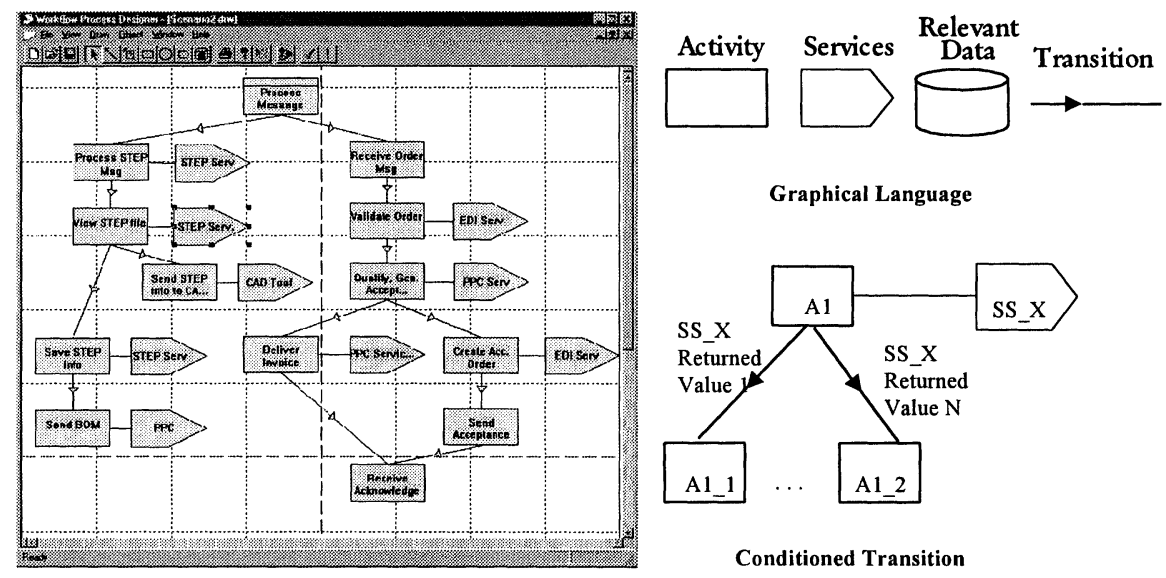

Figure 5 Graphical Workflow Editor.

The Company Profile represents the information about a company itself, which means information about the characteristics of the company's internal environment (such as the engineering systems), about the PCL users inside the company, and the customization of the PCL configurable "features".

The PCL Workflow Model (PWM) defines the customized way under which PCL has to handle the incoming messages. For instance, what are the actions to be taken by PCL when a client order arrives. In other words, it defines how a company is going to use PCL services. PWM is based on the $\mathrm{WfMC}^{3}$ reference model. A

\footnotetext{
${ }^{3}$ Workflow Management Coalition Group, a well-known attempt towards the standardization of the Workflow Management Systems.
} 
workflow model editor was developed, using a graphical language and WPDL syntactic/semantic rules were adopted to represent the entities defined in the WfMC reference model (Figure 5). PWM is a graph where each branch corresponds to a certain PCL service.

The VE Network Directory contains the information about the VE environment itself. In other words, it contains all information about each VE the company is involved in, since a company is able to participate in several VEs simultaneously. It has two parts (Figure 6): the VE General Information and the Partners Directory Information. The first part has the general information about a VE itself. The second part has the information about each partner the company has relationships with, inside that VE.

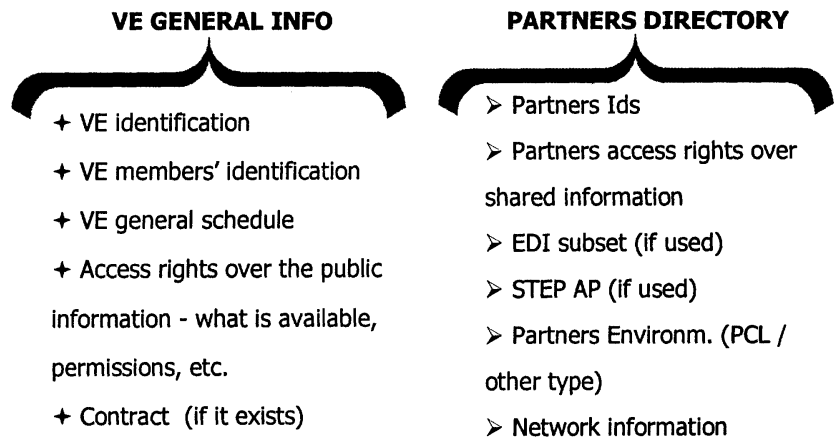

Figure 6 VE Network Directory structure.

Some data must be exchanged during the VE configuration phase. For instance, if two companies exchange EDI messages in a VE, there should be only one common EDI subset being used by both of them. PCL provides a special EDI editor to support the configuration of that EDI subset. To avoid errors and duplicated efforts, the subset definition will be made at one company and it will be sent to the other one.

\subsection{Separating Data \& Control inside PCL}

One important aspect related to the PCL performance is a clear separation between the message contents and the mechanisms to control the message flow inside PCL. As mentioned before, LCM is the module responsible for the coordination among PCL modules, and DIMS stores all data handled by PCL. In order to provide a suitable separation between data and control, LCM and DIMS modules have to work in a strong connection. The data part itself (message content) is stored in the DIMS while LCM deals with control using only a key reference to that data.

PCL internal messages can be classified according to the message purpose. There are control messages and the data messages. A control message is used just for control purposes, i.e., it has only control information. A data message is used to store/retrieve data to/from DIMS. A data message can involve a large amount of data, depending on the type of message. A natural consequence of this 
classification is that LCM only deals with control messages whilst DIMS only deals with data messages.

In order to illustrate what happens when a message arrives at PCL, it is necessary to introduce a new module: the PCI. PCI stands for PRODNET Communication Infrastructure. It is the module responsible for handling the communication services, which involves also the privacy/authentication mechanisms. At this level, PCI is only concerned with sending/receiving messages from both internal company module and VE environment sides. As mentioned above, PCI has adopted international standards related to communication protocols and privacy/authentication algorithms.

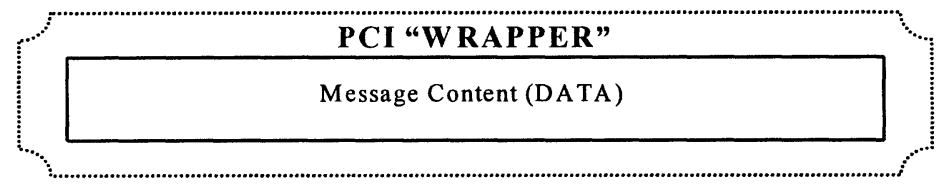

Figure 7 Message structure

A message arriving at PCL has the general structure presented in Figure 7. There are two major parts: the "PCI wrapper" and the message content. The "PCL wrapper" identifies a message as a PCL message and contains the relevant information to be used by PCI privacy/authentication procedures. The message content is the data part itself.

Figure 8 presents an illustrative example to show how a message is handled inside PCL: a client order arrives and PCI receives it. PCI removes the wrapper, applies the privacy/authentication procedures and send it to the LCM. On its turn, LCM stores the message content (data itself) in DIMS and triggers the appropriate PWM flow of events to process that message. All modules involved in that flow may use the data part stored in DIMS, according to their participation in the workflow plan.

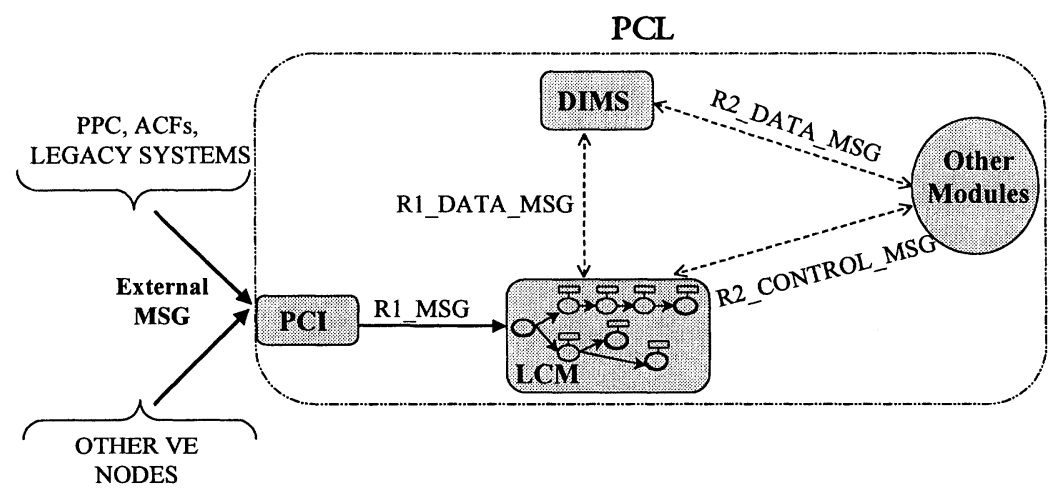

Figure 8 Message handling inside PCL $\left(1^{\text {st }}\right.$ scenario $)$ 
However, considering that PCI receives all messages coming from the external world, in order to guarantee both authentication and safety of the messages, this full cycle should be applied only to messages coming from VE partners; the internal messages could go directly to LCM.

Thus, there is a strong difference between the messages coming from the external world and the internal side of the company. No authentication/safety problems are expected from the messages coming from PPC and other internal legacy systems. Thus, an alternative and optimized scenario is presented in Figure 9. PCI receives only the messages coming from VE partners whilst LCM receives directly the messages coming from the internal side of the company. Internal format means PCIP messages. External format means PECP messages. The separation between control and data management follows the same principles described above.

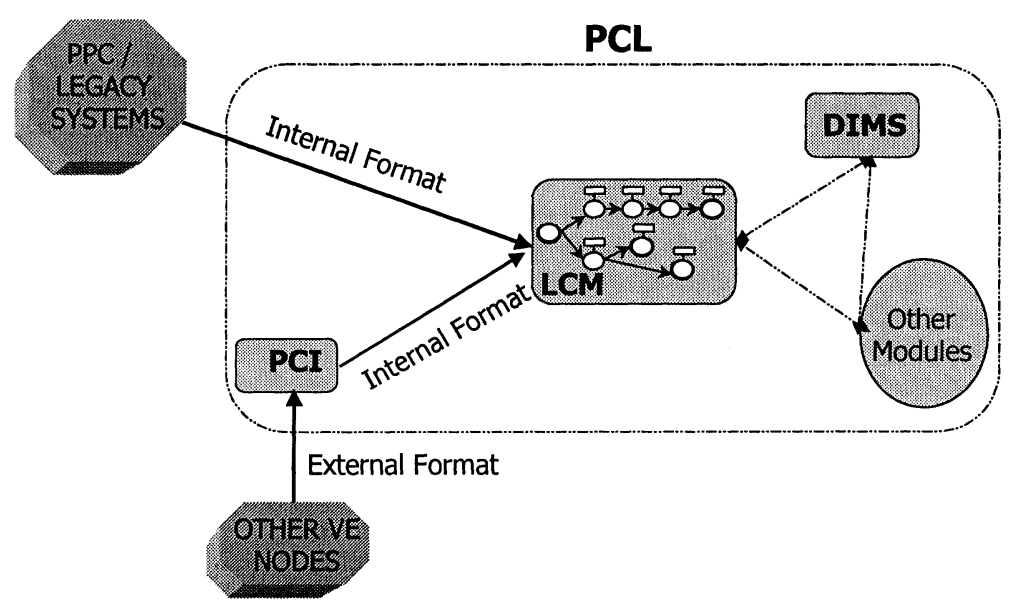

Figure 9 Message handling inside PCL

\subsection{Workflow execution and monitoring}

PCL relies on the LCM module to provide the workflow management services. LCM is split in two logical components: LCM kernel and LCM monitor. LCM kernel is the executor of the PWM, which means it handles the events associated to a VE environment according to the PWM definition. LCM monitor allows both visualization of and interaction with the LCM kernel during the execution of a PWM. Through this monitor, a human operator can: 
- Visualize all messages coming to the company and sent by the VE partners. In other words, s/he can trace all services execution inside PCL;

- Answer requests from the LCM kernel (for instance, what to do with unexpected events);

- Abort a service execution.

As mentioned before, a PWM branch is a sequence of activities. In order to provide a good monitoring environment during the PWM execution, the monitor uses different colors to indicate the execution status of each activity of each branch. The activities status are inactive (gray), active (light green), suspended (light blue) and pre-conditioned (magenta). Thus, the human operator can know exactly what is happening inside PCL: what events are being executed and what is the execution status of each one. For example, if one activity has been suspended for a long time, he/she can decide to abort that service.

\section{CONCLUSIONS AND OPEN QUESTIONS}

The field of Virtual Enterprises represent a very challenging application area for cooperative systems approaches and a new way of organizing manufacturing systems. The architecture being developed by the Esprit PRODNET II project addresses some of these challenges, offering an open environment for cooperation between autonomous enterprises. This paper discussed some aspects related to the information flows, including the identification of various classes of information that need to be exchanged between members of the VE and a flexible approach to coordinate these flows.

PRODNET II has found strong similarities between VE configuration I management and the functionalities of a workflow system (Camarinha-Matos, 1997c). Furthermore, a workflow management approach facilitates the separation between services and coordination / control, facilitating the implementation of a flexible coordination environment. Flexible coordination is a requirement to cope with the diversity of VE organizations and the evolving nature of the cooperation and trust level between enterprises.

The complexity of the VE area and its large multidisciplinarity, that involves not only advanced IT aspects but also the social, organizational and legal issues, raises a lot of important challenges that demand further research. One such challenge is the design of a hierarchical coordination approach (Camarinha-Matos, 1998). On one hand, there are two clearly distinct levels: the global VE coordination level and the coordination of the cooperation events inside each member enterprise. On the other hand, even inside a single VE member, there are a large number of cooperation events to be handled, which require the definition of several subworkflow plans. In order to facilitate the understanding and management of such plans the definition of a hierarchy of coordination levels seems adequate. In the current implementation, PRODNET II considers three levels. 
At the very first level, the PCL services are bounded by the PCL itself. In other words, the support services providers are the PCL modules only. In the second level, the PCL user services are bounded by the company. In other words, the legacy systems are considered as support service providers. In the third level, the PCL services are bounded by the whole VE. In this case, the VE partners (through their legacy systems) are considered as support service providers.

The interactions between the various levels of this hierarchical coordination and the definition of the support services for each level is a topic of ongoing research.

\section{Acknowledgments}

The authors would like to thanks the PRODNET II consortium, and the support from both the European Commission and the Brazilian governmental research funding agency $(\mathrm{CNPq})$.

\section{REFERENCES}

Camarinha-Matos, L.M.; Carelli, R.; Pellicer, J.; Martin, M. - Towards the virtual enterprise in the food industry, ISIP'97 - OE/IEEE/IFIP Int. Conf. on Integrated and Sustainable Industrial Production, Lisboa, May 1997 (a).

Camarinha-Matos, L.M.; H. Afsarmanesh, C. Garita, C. Lima - Towards an Architecture for Virtual Enterprises, The Second World Congress on Intelligent Manufacturing Processes \& Systems, Budapest, Hungary, June 10-13, 1997 (b).

Camarinha-Matos, L.M.;C. Lima, L. Osório, The PRODNET platform for production planning and management in virtual enterprises. Proceedings of ICE'97 $4^{\text {th }}$ International Conference on Concurrent Engineering, Nottingham, October 8-10, 1997 (c).

Camarinha-Matos, L. M.; H. Afsarmanesh, Cooperative Systems challenges in virtual enterprises. Proceedings of CESA'98 Conference, Hammamet, Tunisia, April 1998.

Afsarmanesh, H.; C. Garita, L O Hertzberger, V. Santos Silva, Management of distributed information in Virtual Enterprises. Proc. of ICE' $974^{\text {th }}$ International Conference on Concurrent Engineering, Nottingham, October 8-10, 1997 (a).

H. Afsarmanesh (UVA), L.M. Camarinha-Matos (UNL) Federated Information Management for Cooperative Virtual Organizations, Proceedings of DEXA'97 Int. Conf. On Data Bases and Expert Systems Applications (Lecture Notes in Computer Science, Springer-Verlag), Toulouse, Sep 97 (b).

EAN 1996 - EANCOM, an International Standard for EDI Communication. Available at http://www.aliis.belean/html/EANtCom.html.

\section{BIOGRAPHY}

Dr. Luis Camarinha-Matos is associate professor at the New University of Lisbon where he coordinates the Robotics and CIM group. He has been involved, both as researcher and as technical coordinator, on several international research projects in the areas of virtual enterprises, multiagent systems, intelligent manufacturing systems and machine learning. He has served in the Program Committee of many conferences and was one of the founders of the BASYS conferences series. 
Celson P. Lima received his degree in Computer Science in 1986 and his Master degree in Mechanical Engineering in 1994 from Federal University of Santa Catarina, Brazil. He is currently pursuing his $\mathrm{Ph} . \mathrm{D}$. from the Electrical Engineering Department of the New University of Lisbon. His main interests are: Virtual Enterprises, Workflow systems, EDI and Electronic Commerce. 